\title{
Troponin T in COVID-19 hospitalized patients: Kinetics matter
}

\author{
Maria-Luiza Luchian ${ }^{1}$, Andreea Iulia Motoc ${ }^{1}$, Stijn Lochy ${ }^{1}$, Julien Magne ${ }^{2}$, \\ Bram Roosens ${ }^{1}$, Dries Belsack ${ }^{3}$, Karen Van den Bussche ${ }^{1}$, Berlinde von Kemp ${ }^{1}$, \\ Xavier Galloo ${ }^{1}$, Clara François ${ }^{1}$, Esther Scheirlynck ${ }^{1}$, Sven Boeckstaens ${ }^{1}$, \\ Tom De Potter ${ }^{4}$, Lucie Seyler ${ }^{5}$, Johan van Laethem ${ }^{5}$, Sophie Hennebicq ${ }^{6}$, \\ Caroline Weytjens ${ }^{1}$, Steven Droogmans ${ }^{1}$, Bernard Cosyns ${ }^{1}$ \\ ${ }^{1}$ Department of Cardiology, Vrije Universiteit Brussel (VUB), Universitair Ziekenhuis Brussel, \\ (Centrum voor Hart- en Vaatziekten), Brussels, Belgium \\ ${ }^{2} \mathrm{CHU}$ Limoges, Hôpital Dupuytren, Service Cardiologie, Limoges, France \\ ${ }^{3}$ Department of Radiology, Vrije Universiteit Brussel (VUB), \\ Universitair Ziekenhuis Brussel, Brussels, Belgium \\ ${ }^{4}$ Faculty of Medicine and Pharmacy, Vrije Universiteit Brussel (VUB), Brussels, Belgium \\ ${ }^{5}$ Department of Internal Medicine and Infectious Diseases, Vrije Universiteit Brussel (VUB), \\ Universitair Ziekenhuis Brussel, Brussels, Belgium \\ ${ }^{6}$ Department of Nephrology, University Hospital of Ambroise Pare, Mons, Belgium
}

\begin{abstract}
Background: Coronavirus disease 2019 (COVID-19) emerged as a worldwide health crisis, overwhelming healthcare systems. Elevated cardiac troponin $T$ ( $c T n T)$ at admission was associated with increased in-hospital mortality. However, data addressing the role of $c$ Tn $T$ in major adverse cardiovascular events (MACE) in COVID-19 are scarce. Therefore, we assessed the role of baseline cTn T and cTn T kinetics for MACE and in-hospital mortality prediction in COVID-19.

Methods: Three hundred and ten patients were included prospectively. One hundred and eight patients were excluded due to incomplete records. Patients were divided into three groups according to $c T n T$ kinetics: ascending, descending, and constant. The cTn T slope was defined as the ratio of the cTn $T$ change over time. The primary and secondary endpoints were MACE and in-hospital mortality.

Results: Two hundred and two patients were included in the analysis (mean age $64.4 \pm 16.7$ years, 119 [58.9\%] males). Mean duration of hospitalization was $14.0 \pm 12.3$ days. Sixty (29.7\%) patients had MACE, and 40 (19.8\%) patients died. Baseline c Tn T predicted both endpoints ( $p=0.047$, hazard ratio [HR] 1.805, 95\% confidence interval [CI] 1.009-3.231; $p=0.009$, HR 2.322, 95\% CI 1.234-4.369). Increased cTn T slope predicted mortality ( $p=0.041$, HR 1.006, 95\% CI 1.000-1.011). Constant $c T n$ T was associated with lower MACE and mortality $(p=0.000$, HR 3.080, 95\% CI 1.914-4.954, $p=0.000$, HR 2.851, 95\% CI 1.828-4.447).

Conclusions: The present study emphasizes the additional role of cTn T testing in COVID-19 patients for risk stratification and improved diagnostic pathway and management. (Cardiol J 2021;28, 6: 807-815)

Key words: myocardial injury, cardiac troponin, kinetics, mortality, COVID-19, major cardiovascular adverse events
\end{abstract}

Address for correspondence: Maria Luiza Luchian, MD, Department of Cardiology, Universitair Ziekenhuis Brussel (Centrum voor Hart- en Vaat ziekten), Laerbeeklaan 101, 1090, tel: +32468531461, Brussels, Belgium, e-mail: marialuiza.luchian@uzbrussel.be

Received: 18.06.2021 Accepted: 11.08.2021 Early publication date: 23.09.2021

This article is available in open access under Creative Common Attribution-Non-Commercial-No Derivatives 4.0 International (CC BY-NC-ND 4.0) license, allowing to download articles and share them with others as long as they credit the authors and the publisher, but without permission to change them in any way or use them commercially. 


\section{Introduction}

Coronavirus disease 2019 (COVID-19) emerged as a worldwide health crisis, overwhelming healthcare systems and especially the intensive care units, due to high numbers of critical cases in a short period of time [1, 2]. Initial publications reported elevated cardiac troponin $\mathrm{T}$ (cTn $\mathrm{T}$ ) at admission to be associated with a worse prognosis and increased in-hospital mortality, possibly due to myocardial injury [3, 4]. However, most of these series evaluated one troponin assay and focused on non-cardiovascular adverse events, for example intensive care unit admissions, and the need for ventilation or vasopressor support [5-7]. Current data addresses insufficiently the association between cTn $\mathrm{T}$ and adverse cardiovascular events in COVID-19 patients. By analogy with acute coronary syndromes, the change of cardiac biomarkers levels over time may indicate myocardial injury [8-11], which may develop at any point during hospitalization. Thus, serial cTn T testing might play a key role in the assessment of disease severity [9]. Moreover, it has been suggested that constant values of c Tn T during hospitalization convey an improved prognosis in COVID-19 patients, whereas a continuous increase in cTn $\mathrm{T}$ values implies a worse outcome [9]. However, few data are available to support this hypothesis.

Therefore, we aimed to assess the additional prognostic value of baseline c Tn $\mathrm{T}$ and $\mathrm{cTn} \mathrm{T}$ kinetics in the prediction of major adverse cardiovascular events (MACE) and in-hospital mortality in COVID-19 hospitalized patients.

\section{Methods}

We prospectively included 310 patients admitted to the Universitair Ziekenhuis Brussel (University Hospital of Brussels), Belgium between March 2020 and April 2020. COVID-19 was confirmed by real-time reverse transcription polymerase chain reaction test. One hundred and eight patients with incomplete information on comorbidities, treatment history, lack of systematic evaluation of the clinical status or biomarkers, and/or short period of hospitalization ( $<48 \mathrm{~h}$ in hospital stay) were excluded from the analysis. Clinical information was collected at admission and during hospitalization by the physicians in charge. Data included demographics, medical history, comorbidities, clinical evaluation, laboratory exams, in-hospital treatment, complications, and outcomes. Two hundred and two $(65.1 \%)$ patients had at least two cTn T values assessed during hospitalization, systematically at $24-48$ hours intervals, after hospital admission. cTn T-values $>0.011 \mu \mathrm{g} / \mathrm{L}$ were considered elevated, according to hospital laboratory cut-offs. cTn $\mathrm{T}$ evolution was analyzed by calculating the slope of change as the ratio of the cTn $\mathrm{T}$ change and the change in time $(\Delta c \mathrm{Tn} \mathrm{T} / \Delta$ time $) . \Delta c \mathrm{Tn} \mathrm{T}$ was defined as the difference between the highest value of cTn T during hospitalization and the baseline value. Baseline cTn T was considered the first cTn T assessment in the first 48 hours after hospital admission.

Patients were divided into three groups according to their cTnT kinetics profile, as follows: group 1 representing ascending $\mathrm{cTn} \mathrm{T}$ profile, formed by patients with a variation of $\mathrm{cTn}$ T values from admission to discharge, with increased slope $(\Delta c \mathrm{Tn}$ $\mathrm{T} / \Delta$ time $\geq 1$ ); group 2 , descending c $\mathrm{Tn} \mathrm{T}$ profile included patients with a decrease of $\mathrm{c} T \mathrm{n} \mathrm{T}$ values from admission to discharge $(\Delta \mathrm{Tn} \mathrm{T} / \Delta$ time $<1)$; and group 3 , constant $\mathrm{cTn} \mathrm{T}$ profile, included patients with a stable value of $\mathrm{cTn}$ T during hospitalization $(\Delta \mathrm{cTn} \mathrm{T} / \Delta$ time $=0)$. MACE was considered as the primary endpoint, composed of all-cause mortality, acute heart failure, acute coronary syndrome, pericarditis, myocarditis, atrial fibrillation or flutter, and pulmonary embolism. In-hospital mortality was considered as the secondary endpoint. All cardiovascular events were defined according to current practice guidelines of cardiology [11-15].

\section{Ethical approval}

This study was approved by the local Ethical Committee and was carried out in accordance with the ethical principles for medical research involving human subjects established by the Helsinki Declaration, protecting the privacy of all participants, as well as the confidentiality of their personal information.

\section{Statistical analyses}

Descriptive statistics were performed for all study variables. Continuous variables were expressed as mean \pm standard deviation (SD) or median (interquartile [IQR]) for skewed variables. Categorical variables were expressed as percentages. Normality of data was tested using the Kolmogorov-Smirnov test. Comparison of continuous variables was performed using Student's t-test or the Mann-Whitney U-test, and of binominal variables using the $\chi^{2}$ or Fisher exact test, respectively. Comparison between three groups was performed using one-way ANOVA for continuous data. Univariable and multivariable Cox regression models 
Table 1. Cox regression analysis for major adverse cardiovascular events (MACE) and for in-hospital mortality in COVID-19 patients for baseline cardiac troponin T (cTn T).

\begin{tabular}{|c|c|c|c|c|c|c|}
\hline \multirow[t]{2}{*}{ Parameter } & \multicolumn{3}{|c|}{ Univariable analysis } & \multicolumn{3}{|c|}{ Multivariable analysis } \\
\hline & HR & $95 \% \mathrm{Cl}$ & $\mathbf{P}$ & HR & $95 \% \mathrm{Cl}$ & $\mathbf{P}$ \\
\hline \multicolumn{7}{|l|}{ MACE predictors } \\
\hline Age & 1.056 & $1.032-1.081$ & 0.000 & 1.054 & $1.025-1.084$ & 0.000 \\
\hline cTn $T$ & 1.966 & $1.225-3.154$ & 0.005 & 1.805 & $1.009-3.231$ & 0.047 \\
\hline Neutrophil-lymphocyte ratio & 1.059 & $1.025-1.093$ & 0.001 & 0.997 & $0.955-1.040$ & 0.877 \\
\hline C-reactive protein & 1.004 & $1.002-1.007$ & 0.002 & 1.004 & $1.001-1.007$ & 0.021 \\
\hline History of diabetes mellitus & 1.232 & $0.689-2.201$ & 0.482 & 1.238 & $0.276-5.551$ & 0.780 \\
\hline History of arterial hypertension & 1.502 & $0.864-2.614$ & 0.150 & 0.889 & $0.428-1.847$ & 0.752 \\
\hline History of dyslipidemia & 1.194 & $0.689-2.071$ & 0.527 & 0.894 & $0.202-3.968$ & 0.883 \\
\hline \multicolumn{7}{|l|}{ In-hospital mortality } \\
\hline Age & 1.069 & $1.040-1.098$ & 0.000 & 1.061 & $1.029-1.094$ & 0.000 \\
\hline Neutrophil-lymphocyte ratio & 1.069 & $1.034-1.106$ & 0.000 & 1.003 & 0.958-1.049 & 0.911 \\
\hline C-reactive protein & 1.005 & $1.002-1.008$ & 0.001 & 1.007 & $1.003-1.012$ & 0.000 \\
\hline cTn T & 2.138 & $1.338-3.416$ & 0.001 & 2.322 & $1.234-4.369$ & 0.009 \\
\hline History of diabetes mellitus & 1.320 & $0.688-2.532$ & 0.404 & 0.845 & $0.383-1.864$ & 0.676 \\
\hline
\end{tabular}

$\mathrm{Cl}$ - confidence interval; $\mathrm{HR}$ - hazard ratio

were used to evaluate potential predictors of inhospital mortality and MACE.

Kaplan-Meier survival curves were used to compare event-free and survival rates for COVID-19 patients by the log-rank test. Receiver operating characteristics (ROC) curves were constructed to determine the ability of baseline cTn T to predict mortality and MACE and to identify its sensitivity and specificity.

Data were analyzed using IBM SPSS Statistic for Windows, Version 26.0 (Armonk, NY: IBM Corp.). A p value $<0.05$ was considered significant.

\section{Results}

\section{Global population: Baseline characteristics}

Two hundred and two patients were included in the analysis (mean age $64.4 \pm 16.7$ years, 119 [58.9\%] males). The mean duration of hospitalization was $14.0 \pm 12.3$ days.

The prevalence of cardiovascular risk factors was the following: hypertension in $102(50.5 \%)$ patients, dyslipidemia in $83(41.1 \%)$ patients, diabetes mellitus in $61(30.2 \%)$ patients, and obesity in $56(27.9 \%)$ patients. Chronic kidney disease was observed in 29 (14.4\%) patients.

Baseline characteristics of the global study population and the comparison between patients with and without primary and secondary endpoints are shown in Supplemental Material Table 1.
Clinical outcomes of the main population

Major adverse cardiovascular events occurred in $60(29.7 \%)$ patients. Twenty-three $(11.4 \%)$ patients developed acute heart failure, $5(2.6 \%)$ patients developed acute coronary syndrome ( 1 acute myocardial infarction with ST segment elevation, 2 patients with acute myocardial infarction without ST segment elevation and 2 Takotsubo syndrome), $4(2 \%)$ patients developed pericarditis, $4(2 \%)$ developed myocarditis, and 3 (1.3\%) developed pulmonary embolism. Fourteen $(6.9 \%)$ patients had acute onset of atrial fibrillation, and $1(0.5 \%)$ patient had atrial flutter.

The in-hospital mortality rate was $40(19.8 \%)$ patients.

Factors associated with MACE and in-hospital mortality in the main population

Baseline cTn T. Univariable and multivariable Cox regression analyses for MACE and in-hospital mortality are shown in Table 1 . Baseline cTn T independently predicted MACE $(\mathrm{p}=0.047$, hazard ratio [HR] 1.805, 95\% confidence interval [CI] 1.009-3.231) and in-hospital mortality $(\mathrm{p}=0.009$, HR 2.322, 95\% CI 1.234-4.369).

For MACE, sensitivity and specificity of baseline cTn T (best cut-off value $0.014 \mu \mathrm{g} / \mathrm{mL}$ ) were $92.6 \%$ and $60.1 \%$, respectively, with an area under the curve of 0.80 (95\% CI 0.73-0.87) (Fig. 1A), and for in-hospital mortality: sensitivity $77.1 \%$ 


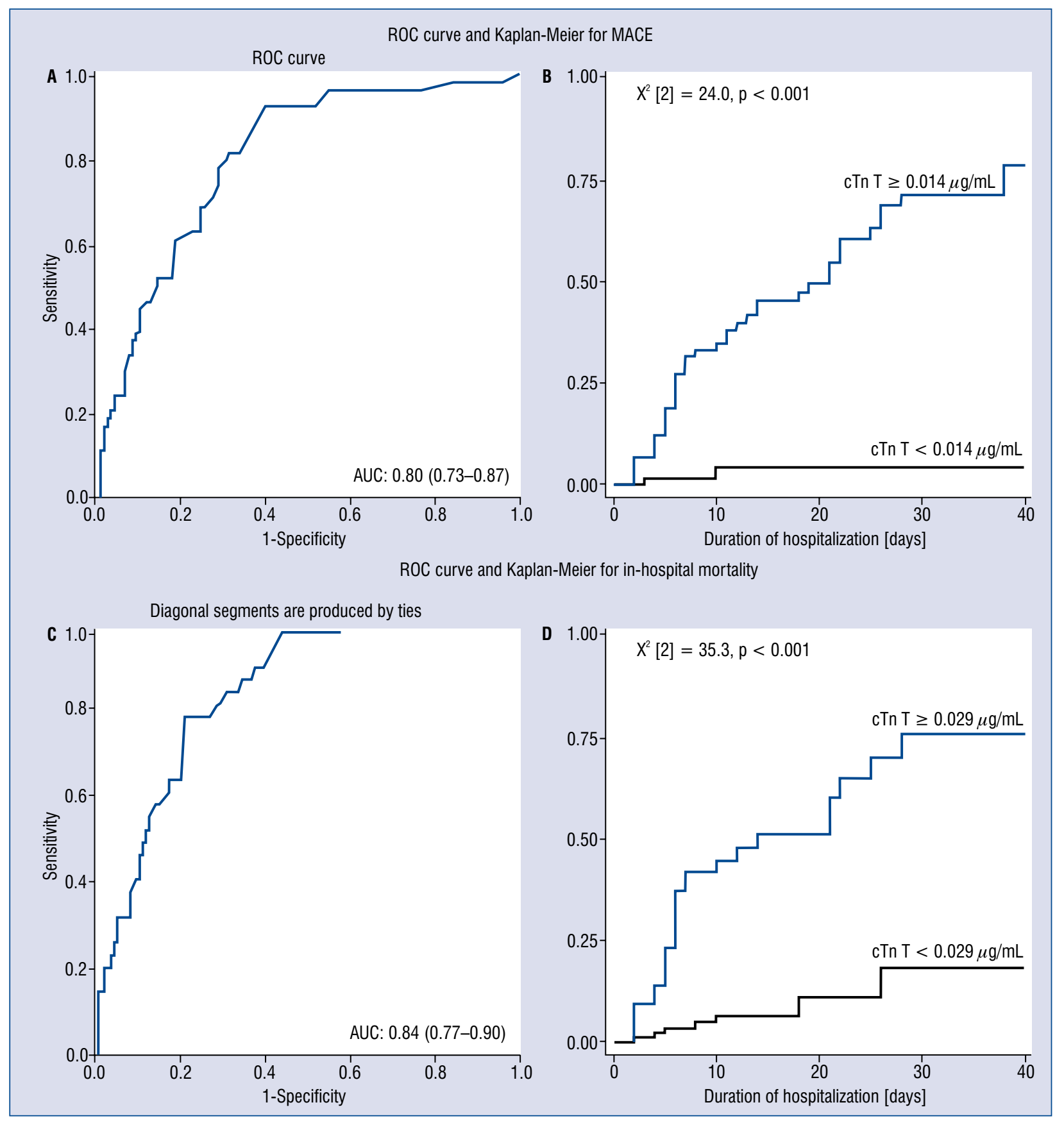

Figure 1. Receiver operating characteristics (ROC) and Kaplan-Meier analysis illustrating the role of baseline cardiac troponin T (cTn T) in major adverse cardiovascular events (MACE) and in-hospital mortality in COVID-19 patients. ROC analysis for baseline cTn T for predicting MACE (A) and in-hospital mortality (C). Kaplan-Meier survival curve analysis for MACE, Log Rank (Matel-Cox) value $=0.000$ (B) and in-hospital mortality, Log Rank (Matel-Cox) value $=0.000$ (D).

and specificity $78.8 \%$; area under the curve 0.84 (95\% CI $0.77-0.90$ ); and best cut-off value 0.029 $\mu \mathrm{g} / \mathrm{mL}$ (Fig. 1C).

Kaplan-Meier survival analysis for MACE and in-hospital mortality showed significant differences between patients with a baseline cTn T more than
$0.014 \mu \mathrm{g} / \mathrm{mL}$ for MACE and more than $0.029 \mu \mathrm{g} / \mathrm{mL}$ for in-hospital mortality $\left(\mathrm{X}^{2}[2]=24.0, \mathrm{p}<0.001\right.$, $\mathrm{X}^{2}[2]=35.3, \mathrm{p}<0.001$, respectively, Fig. $\left.1 \mathrm{~B}, \mathrm{D}\right)$.

cTn T kinetics. Characteristics of groups according to cTn T kinetics are detailed in Supplemental Material Table 2. 
Table 2. Cox regression analysis for major adverse cardiovascular events (MACE) and for in-hospital mortality in COVID-19 patients with constant cardiac troponin T (cTn T) profile and Cox regression analysis for in-hospital mortality in COVID-19 patients with an ascending cTn T slope.

\begin{tabular}{|c|c|c|c|c|c|c|}
\hline \multirow[t]{2}{*}{ Parameter } & \multicolumn{3}{|c|}{ Univariable analysis } & \multicolumn{3}{|c|}{ Multivariable analysis } \\
\hline & HR & $95 \% \mathrm{Cl}$ & $\mathbf{P}$ & HR & $95 \% \mathrm{Cl}$ & $\mathbf{P}$ \\
\hline \multicolumn{7}{|c|}{ MACE predictors in COVID-19 patients with constant cTn T profile } \\
\hline Age & 0.891 & $0.969-0.992$ & 0.001 & 0.994 & $0.979-1.008$ & 0.402 \\
\hline C-reactive protein & 0.998 & $0.996-1.000$ & 0.103 & 1.000 & $0.998-1.003$ & 0.805 \\
\hline Neuthropil-lymphocyte ratio & 0.964 & $0.933-0.997$ & 0.035 & 1.011 & $0.969-1.054$ & 0.615 \\
\hline Constant cTn T profile & 3.133 & $2.140-4.589$ & 0.000 & 3.080 & $1.914-4.954$ & 0.000 \\
\hline History of diabetes mellitus & 0.573 & $0.383-0.857$ & 0.007 & 0.337 & $0.183-0.776$ & 0.008 \\
\hline History of arterial hypertension & 0.835 & $0.576-1.211$ & 0.341 & 1.107 & $0.719-1.703$ & 0.646 \\
\hline History of dyslipidemia & 0.690 & $0.469-1.014$ & 0.059 & 1.766 & $0.884-3.526$ & 0.107 \\
\hline \multicolumn{7}{|c|}{ In-hospital mortality in COVID-19 patients with constant $\mathrm{cTn}$ T profile } \\
\hline Age & 0.983 & $0.972-0.994$ & 0.003 & 0.999 & $0.986-1.012$ & 0.854 \\
\hline C-reactive protein & 0.999 & $0.997-1.001$ & 0.154 & 1.000 & $0.998-1.002$ & 0.991 \\
\hline Neutrophil-lymphocyte ratio & 0.967 & $0.937-0.999$ & 0.041 & 1.006 & $0.968-1.045$ & 0.769 \\
\hline Constant cTn T profile & 3.076 & $2.131-4.440$ & 0.000 & 2.851 & $1.828-4.447$ & 0.000 \\
\hline History of diabetes mellitus & 0.598 & $0.408-0.877$ & 0.009 & 0.690 & $0.462-1.030$ & 0.070 \\
\hline \multicolumn{7}{|c|}{ In-hospital mortality in COVID-19 patients with an ascending cTn T slope } \\
\hline Age & 0.983 & $0.972-0.994$ & 0.003 & 1.100 & $1.017-1.190$ & 0.018 \\
\hline C-reactive protein & 0.999 & $0.997-1.001$ & 0.154 & 1.007 & $1.000-1.015$ & 0.056 \\
\hline Neutrophil-lymphocyte ratio & 0.967 & $0.937-0.999$ & 0.041 & 1.045 & $0.957-1.142$ & 0.327 \\
\hline Ascending slope & 3.076 & $2.131-4.440$ & 0.000 & 1.006 & $1.000-1.011$ & 0.041 \\
\hline History of diabetes mellitus & 0.598 & $0.408-0.877$ & 0.009 & 0.629 & $0.141-2.811$ & 0.544 \\
\hline
\end{tabular}

$\mathrm{Cl}$ - confidence interval; $\mathrm{HR}$ - hazard ratio

Predictors of MACE and in-hospital mortality according to $\mathrm{c}$ Tn $\mathrm{T}$ kinetics pattern

A constant cTn T profile was independently associated with lower MACE and in-hospital mortality rates comparing to the other two groups $(\mathrm{p}=0.000$, HR 3.080, 95\% CI 1.914-4.954, $\mathrm{p}=0.000$; HR $2.851,95 \%$ CI 1.828-4.447, respectively).

Conversely, a positive ascending slope was an independent predictor of in-hospital mortality ( $\mathrm{p}=0.041$, HR 1.006, 95\% CI 1.000-1.011).

The univariable and multivariable Cox regression analyses for MACE and in-hospital mortality are shown in Table 2.

Kaplan-Meier survival analysis for MACE and in-hospital mortality, showed significant differences between patients with a constant cTn T profile and other cTn $\mathrm{T}$ profiles, $\left(\mathrm{X}^{2}[2]=43.4\right.$, $\mathrm{p}<0.001, \mathrm{X}^{2}[2]=41.9, \mathrm{p}<0.001$, respectively, Fig. 2A, B; 3).

\section{Discussion}

The main findings of this study were the following: 1) baseline cTn T independently predicted MACE and in-hospital mortality in COVID-19 hospitalized patients; 2 ) a constant cTn T profile was independently associated with a lower risk of MACE and in-hospital mortality; and 3) a rapid increase of cTn T during hospitalization was associated with higher mortality rate but not with higher MACE rate in hospitalized COVID-19 patients.

The characteristics of our study population are similar to earlier reports [16]. Patients diagnosed with COVID-19 are older, implicitly more fragile, and have more cardiac risk factors, which predispose them to developing major cardiovascular events [17-19].

Previous studies primarily focused on the role of baseline cTn $\mathrm{T}$ in predicting non-cardiac major 


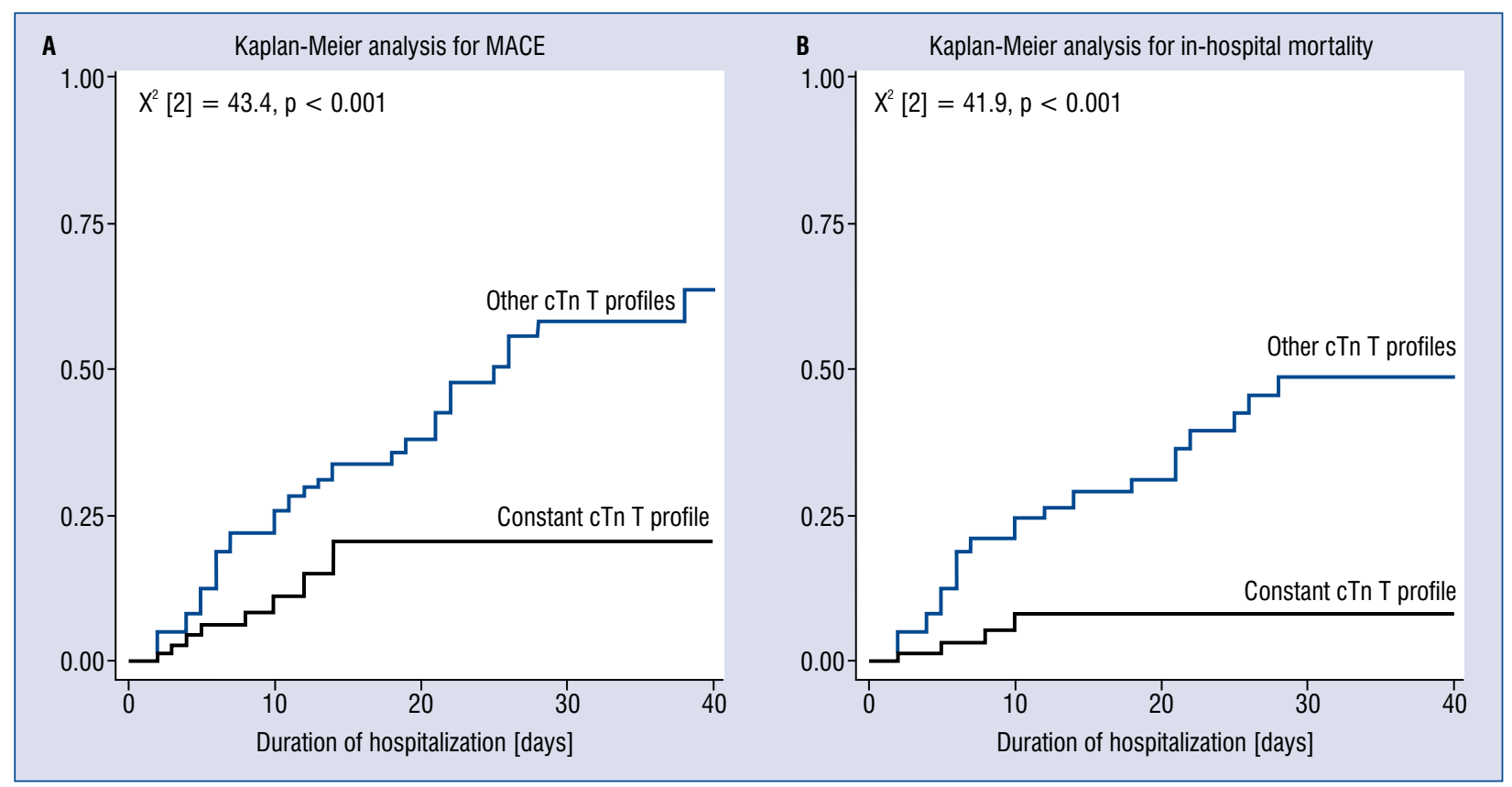

Figure 2. Kaplan-Meier survival analysis on the role of constant troponin profile in major adverse cardiovascular events (MACE) and in-hospital mortality in COVID-19 patients. Kaplan-Meier survival curve analysis for MACE, Log Rank (Matel-Cox) value $=0.000(\mathbf{A})$ and in-hospital mortality, Log Rank (Matel-Cox) value $=0.000$ (B).

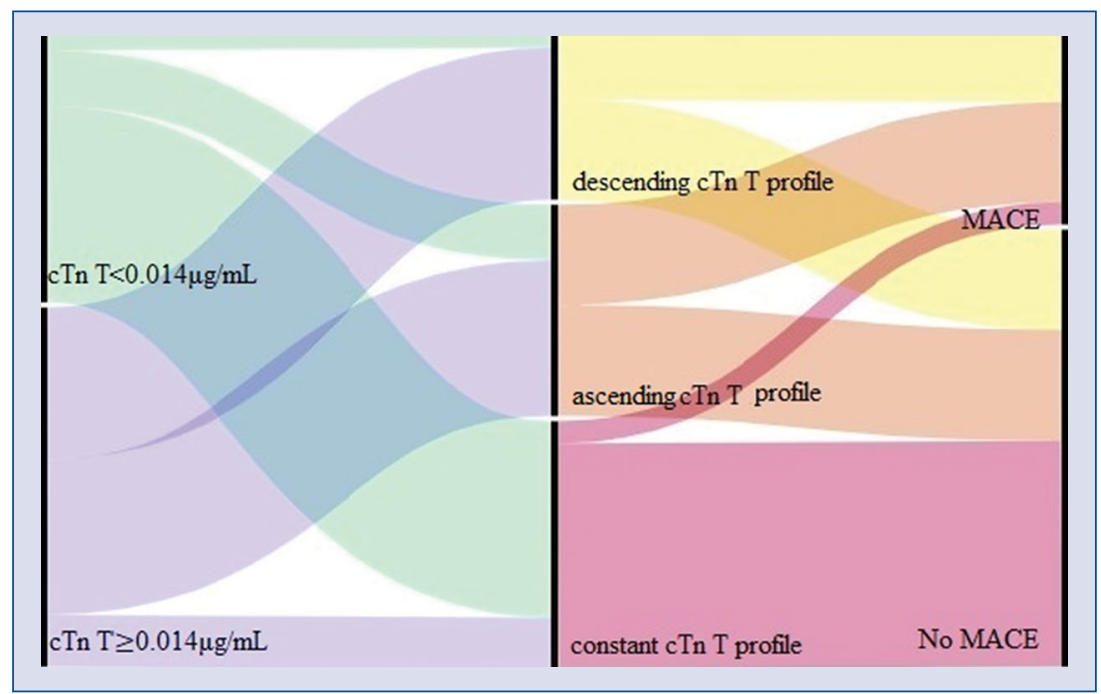

Figure 3. Alluvial plot illustrating the distribution of values of baseline cardiac troponin T (cTn T) in COVID-19 patients divided into three kinetics profiles according on the values during hospitalization of cardiac troponin (ascending cTn T, descending cTn T, and constant cTn T) and the association with major cardiovascular events. First column: patients were divided into two groups based on the best cut-off value of baseline $\mathrm{cTn}$ T of $0.014 \mu \mathrm{g} / \mathrm{mL}$ (green: $\mathrm{cTn} \mathrm{T}<0.014$ $\mu \mathrm{g} / \mathrm{mL}$, purple: $\mathrm{cTn} \mathrm{T} \geq 0.014 \mu \mathrm{g} / \mathrm{mL}$ ), which was shown as an independent predictor for major adverse cardiovascular events (MACE). Second column: patients were separated into three cTn T kinetics profiles: ascending troponin profile (orange), descending troponin profile (yellow), constant troponin profile (pink). Third column: Patients without MACE. Patients with MACE.

events such as intensive care unit admission, need for invasive ventilation or extracorporeal membrane oxygenation, and in-hospital mortality $[6,7$,
10, 20]. Elevated cardiac biomarkers, especially cardiac troponin, were identified in about $10 \%$ to $20 \%$ of hospitalized COVID-19 patients, especially 
in those with a more severe form of the disease and non-survivors [21]. Both cardiovascular comorbidities and high values of cTn $\mathrm{T}$ were correlated to higher morbidity and mortality in COVID-19 patients [21]. However, the small number or even the complete absence of cTn T measurements at baseline and during hospitalization were among the most frequently encountered limitations of earlier reports. Thus, data on the cardiac involvement and potential mechanisms of myocardial injury in COVID-19 are still scarce.

Possible mechanisms attributed to myocardial damage and previously observed in other viral infections such as influenza were: severe inflammation, oxygen mismatch supply-demand, thrombosis, and microvascular injury [6, 8, 22]. This is further supported by recent pathological reports on the presence of severe acute respiratory syndrome-coronavirus- 2 in heart specimens, proving the cardiac tropism [23].

In the current study, elevated troponin levels at baseline were linked not only to higher mortality rates confirming the results of earlier reports [16], but they also independently predicted major cardiovascular events, with a high sensitivity and specificity. These findings emphasize the susceptibility to myocardial injury related to COVID-19 infection.

Sandoval et al. [9] postulated that in addition to baseline cTn T, stable concentrations of cTn T over time convey a lower risk of cardiovascular complications, such as myocarditis, pericarditis, or acute coronary syndromes, whereas a more in-depth approach is advised in patients with an increase in cTn T values in time. The present study reinforces this statement, by showing that a rapid increase of cTn $\mathrm{T}$ over a short period of time was independently associated with inhospital mortality. The increase in cTn T values may reflect the severity of myocardial injury and may be linked to the amplitude of inflammatory response [18, 21, 24].

In our cohort, higher values of C-reactive protein were associated with increased cTn $\mathrm{T}$ values during hospitalization. Moreover, baseline C-reactive protein was significantly associated with both major endpoints, suggesting that inflammation acts as a trigger for acute cardiac damage and increases morbidity and mortality in these patients [25].

These findings are further supported by previous studies on severe acute respiratory syndrome (SARS) and Middle East respiratory syndrome (MERS), which showed that an exaggerated immune response with release of inflammatory cy- tokines may cause myocardial damage with high levels of cardiac biomarkers [26].

Interestingly, patients with constant troponin values during hospitalization had lower rates of death or major cardiovascular events, even though they might have had elevated baseline cTn T.

This finding suggests that the increase in $\mathrm{cTn} T$ at admission might be caused by extra cardiac factors, unrelated to cardiac events, supporting the additional role of serial cTn T testing in these patients [27, 28].

Nevertheless, worse outcomes were also attributed to COVID-19 patients with cardiovascular comorbidities and risk factors, such as arterial hypertension, obesity, or diabetes mellitus [5, 29]. However, in the present study, no significant differences in terms of cardiac risk factors or pathologies between c Tn T groups according to cTn T kinetics were found, except for diabetes mellitus. Nonetheless, it was not linked to higher mortality or major cardiovascular events.

Hence, these results underline that the clinical significance of serial testing in COVID-19 patients should not be overlooked, irrespective of the presence of cardiovascular disease or risk factors. The findings of the present study emphasize the additional role of troponin testing, not only on admission, but also during hospitalization, for a better risk stratification and an improved diagnostic pathway. Moreover, patients with cardiac injury during hospitalization should be routinely monitored for mid- and long-term outcomes, following COVID-19 infection.

\section{Limitations of the study}

This study has several limitations. This is a single-center study with a limited number of patients. It might be argued that cardiac troponin was assessed in more severe forms of COVID-19. Due to logistical restriction at the beginning of the pandemic, some data regarding echocardiography, cardiac magnetic resonance, or systematic measurements of cardiac biomarkers were lacking in some patients. Cardiovascular examinations were performed in selected cases based on the clinical and biological status of the patients, according to current guideline recommendations to avoid the risk of cross-infections [27, 30]. Therefore, serial echocardiographic evaluation was not available in all COVID-19 patients.

Nonetheless, patients who died were more likely to have missing data, including serial cardiac troponin testing. Thus, patients with incomplete data were excluded from the final analysis. 
Also, in the present study less sensitive cardiac troponin assays were used. However, the value of troponin in distinguishing patients with worse outcome was obvious.

Multicenter research on a larger population with a standardized protocol is necessary to confirm our results.

\section{Conclusions}

Elevated cTn $\mathrm{T}$ at admission independently predicted MACE and in-hospital mortality in COVID-19 hospitalized patients. Moreover, a rapid increase in the value of cTn T was associated with higher mortality rates.

Conversely, a constant cTn T profile was linked to lower rates of MACE and in-hospital mortality. These findings emphasize the additional value of serial troponin testing in COVID-19 patients, for risk stratification and improved diagnosis pathways and management.

\section{Conflict of interest: None declared}

\section{References}

1. WHO, Aylward, Bruce (WHO); Liang W (PRC). Report of the WHO-China Joint Mission on Coronavirus Disease 2019 (COVID-19). WHO-China Jt Mission Coronavirus Dis 2019. 2020.

2. Shi S, Qin Mu, Shen Bo, et al. Association of cardiac injury with mortality in hospitalized patients with COVID-19 in Wuhan, China. JAMA Cardiol. 2020; 5(7): 802-810, doi: 10.1001/jamacardio.2020.0950, indexed in Pubmed: 32211816.

3. Shi S, Qin Mu, Cai Y, et al. Characteristics and clinical significance of myocardial injury in patients with severe coronavirus disease 2019. Eur Heart J. 2020; 41(22): 2070-2079, doi: 10.1093/eurheartj/ehaa408, indexed in Pubmed: 32391877.

4. Kuno T, Takahashi M, Obata R, et al. Cardiovascular comorbidities, cardiac injury, and prognosis of COVID-19 in New York City. Am Heart J. 2020; 226: 24-25, doi: 10.1016/j.ahj.2020.05.005, indexed in Pubmed: 32425197.

5. Guan Wj, Liang Wh, Zhao Yi, et al. Comorbidity and its impact on 1,590 patients with COVID-19 in China: A Nationwide Analysis. Eur Respir J. 2020, doi: 10.1101/2020.02.25.20027664.

6. Guo T, Fan Y, Chen M, et al. Cardiovascular Implications of Fatal Outcomes of Patients With Coronavirus Disease 2019 (COVID-19). JAMA Cardiology. 2020; 5(7): 811, doi: 10.1001/ jamacardio.2020.1017.

7. Sabatino J, De Rosa S, Di Salvo G, et al. Impact of cardiovascular risk profile on COVID-19 outcome. A meta-analysis. PLoS One. 2020; 15(8): e0237131, doi: 10.1371/journal.pone.0237131, indexed in Pubmed: 32797054.

8. Lala A, Johnson KW, Januzzi JL, et al. Prevalence and Impact of Myocardial Injury in Patients Hospitalized with COVID-19 Infection. medRxiv. 2020; 76(5): 533-546, doi: 10.1101/2020.04.20.20072702, indexed in Pubmed: 32511658.
9. Sandoval Y, Januzzi JL, Jaffe AS. Cardiac troponin for assessment of myocardial injury in COVID-19: JACC review topic of the week. J Am Coll Cardiol. 2020; 76(10): 1244-1258, doi: 10.1016/j. jacc.2020.06.068, indexed in Pubmed: 32652195.

10. Lorente-Ros A, Monteagudo Ruiz JM, Rincón LM, et al. Myocardial injury determination improves risk stratification and predicts mortality in COVID-19 patients. Cardiol J. 2020; 27(5): 489-496, doi: 10.5603/CJ.a2020.0089, indexed in Pubmed: 32589258.

11. Collet JP, Thiele H. 2020 ESC Guidelines for the management of acute coronary syndromes in patients presenting without persistent ST-segment elevation. Eur Heart J. 2020; 41(37): 3495-3497, doi: 10.1093/eurheartj/ehaa624.

12. Meguro S, Ishibashi M, Takei I. [The significance of high sensitive $\mathrm{C}$ reactive protein as a risk factor for cardiovascular diseases]. Rinsho Byori. 2012; 60(4): 356-361, indexed in Pubmed: 22686046.

13. Yancy C, Jessup M, Bozkurt B, et al. 2017 ACC/AHA/HFSA Focused Update of the 2013 ACCF/AHA Guideline for the Management of Heart Failure. J Cardiac Failure. 2017; 23(8): 628-651, doi: 10.1016/j.cardfail.2017.04.014.

14. Konstantinides SV, Meyer G, Becattini C, et al. ESC Scientific Document Group, The Task Force for the diagnosis and management of acute pulmonary embolism of the European Society of Cardiology (ESC). 2019 ESC Guidelines for the diagnosis and management of acute pulmonary embolism developed in collaboration with the European Respiratory Society (ERS): The Task Force for the diagnosis and management of acute pulmonary embolism of the European Society of Cardiology (ESC). Eur Heart J. 2020; 41(4): 543-603, doi: 10.1093/eurheartj/ehz405, indexed in Pubmed: 31504429.

15. Brugada J, Katritsis DG, Arbelo E, et al. 2019 ESC Guidelines for themanagement of patients with supraventricular tachycardia. Eur Heart J. 2020; 41(5): 655-720, doi: 10.1093/eurheartj/ ehz467, indexed in Pubmed: 31504425.

16. Majure DT, Gruberg L, Saba SG, et al. Usefulness of elevated troponin to predict death in patients with COVID-19 and myocardial injury. Am J Cardiol. 2021; 138: 100-106, doi: 10.1016/j. amjcard.2020.09.060, indexed in Pubmed: 33058800.

17. $\mathrm{Xu} \mathrm{H}, \mathrm{Hou} \mathrm{K}, \mathrm{Xu}$ R, et al. Clinical characteristics and risk factors of cardiac involvement in COVID-19. J Am Heart Assoc. 2020; 9(18): e016807, doi: 10.1161/JAHA.120.016807, indexed in Pubmed: 32806998.

18. Raad M, Dabbagh M, Gorgis S, et al. Cardiac injury patterns and inpatient outcomes among patients admitted with COVID-19. Am J Cardiol. 2020; 133: 154-161, doi: 10.1016/j.amjcard.2020.07.040, indexed in Pubmed: 32829913.

19. Núñez-Gil IJ, Fernández-Ortiz A, Maroud Eid C, et al. Underlying heart diseases and acute COVID-19 outcomes. Cardiol J. 2021; 28(2): 202-214, doi: 10.5603/CJ.a2020.0183, indexed in Pubmed: 33346365.

20. Deng Q, Hu Bo, Zhang Y, et al. Suspected myocardial injury in patients with COVID-19: Evidence from front-line clinical observation in Wuhan, China. Int J Cardiol. 2020; 311: 116-121, doi: 10.1016/j.ijcard.2020.03.087, indexed in Pubmed: 32291207.

21. Mueller C, Giannitsis E, Jaffe AS, et al. Cardiovascular biomarkers in patients with COVID-19. Eur Heart J Acute Cardiovasc Care. 2021; 10(3): 310-319, doi: 10.1093/ehjacc/zuab009, indexed in Pubmed: 33655301.

22. Chow EJ, Rolfes MA, O'Halloran A, et al. Acute cardiovascular events associated with influenza in hospitalized adults: a cross- 
sectional study. Ann Intern Med. 2020; 173(8): 605-613, doi: 10.7326/M20-1509, indexed in Pubmed: 32833488.

23. Pesaresi M, Pirani F, Tagliabracci A, et al. SARS-CoV-2 identification in lungs, heart and kidney specimens by transmission and scanning electron microscopy. Eur Rev Med Pharmacol Sci. 2020; 24(9): 5186-5188, doi: 10.26355/eurrev_202005_21217, indexed in Pubmed: 32432787.

24. Giustino G, Croft L, Stefanini G, et al. Characterization of myocardial injury in patients with COVID-19. J Am Coll Cardiol. 2020; 76(18): 2043-2055, doi: 10.1016/j.jacc.2020.08.069.

25. Li JJ, Fang CH. C-reactive protein is not only an inflammatory marker but also a direct cause of cardiovascular diseases. Med Hypotheses. 2004; 62(4): 499-506, doi: 10.1016/j. mehy.2003.12.014, indexed in Pubmed: 15050096.

26. Park JE, Jung S, Kim A, et al. MERS transmission and risk factors: a systematic review. BMC Public Health. 2018; 18(1): 574, doi: 10.1186/s12889-018-5484-8, indexed in Pubmed: 29716568.
27. Cosyns B, Lochy S, Luchian ML, et al. The role of cardiovascular imaging for myocardial injury in hospitalized COVID-19 patients. Eur Heart J Cardiovasc Imaging. 2020; 21(7): 709-714, doi: 10.1093/ehjci/jeaa136, indexed in Pubmed: 32391912.

28. Mahajan VS, Jarolim P. How to interpret elevated cardiac troponin levels. Circulation. 2011; 124(21): 2350-2354, doi: 10.1161/CIRCULATIONAHA.111.023697, indexed in Pubmed: 22105197.

29. Li X, Guan Bo, Su T, et al. Impact of cardiovascular disease and cardiac injury on in-hospital mortality in patients with COVID-19: a systematic review and meta-analysis. Heart. 2020; 106(15): 1142-1147, doi: 10.1136/heartjnl-2020-317062, indexed in Pubmed: 32461330.

30. Skulstad H, Cosyns B, Popescu BA, et al. COVID-19 pandemic and cardiac imaging: EACVI recommendations on precautions, indications, prioritization, and protection for patients and healthcare personnel. Eur Heart J Cardiovasc Imaging. 2020; 21(6): 592-598, doi: 10.1093/ehjci/jeaa072, indexed in Pubmed: 32242891. 